\title{
BRIEF REPORT Malignant Fibrous Histiocytoma Two Years after Autologous Stem Cell Transplant for Hodgkin Lymphoma: Evidence for Genomic Instability
}

\author{
Shanmuganathan Chandrakasan, MD, ${ }^{1}$ Christine J. Ye, MD, ${ }^{2}$ Meera Chitlur, MD, ${ }^{1}$ Anwar N. Mohamed, MD, ${ }^{3}$ \\ Raja Rabah, MD, ${ }^{3}$ Andre Konski, $\mathrm{MD}^{4}{ }^{4}$ Henry H. Q. Heng, PhD, ${ }^{2}$ and Süreyya Savaşan ${ }^{1,5 *}$
}

Secondary malignancies $(\mathrm{SMs})$ in Hodgkin lymphoma $(\mathrm{HL})$ are thought to be related to exposure to alkalating agents, topoisomerase II inhibitors and ionizing radiation, and tend to occur a decade after initial therapy. We report a 14 year old autistic male, who developed malignant fibrous histiocytoma (MFH) two years after autologous stem cell transplantation for advanced stage HL. The
MFH and post-surgical reactive tissues exhibited multiple clonal abnormalities. In addition, PHA-stimulated peripheral blood lymphocytes showed increased frequency of non-clonal chromosomal aberrations. The potential role of genomic instability in early onset of SM in our patient is discussed. Pediatr Blood Cancer 2011; 56:1143-1145. @ 2011 Wiley-Liss, Inc.

Key words: Autism; Autologous stem cell transplantation; Genomic instability; Hodgkin lymphoma; Malignant fibrous histiocytoma; Non-clonal chromosome aberration (NCCA)

\section{INTRODUCTION}

Hodgkin lymphoma (HL) constitutes $6 \%$ of all childhood cancers. With the use of effective chemotherapy, radiotherapy, and autologous stem cell transplant (ASCT) in recurrent cases, the prognosis has improved dramatically in HL [1]. The increase in survival rate has placed more emphasis on managing long-term morbidity and mortality among the survivors.

Secondary malignancy $(\mathrm{SM})$ is one of the main contributors to long-term morbidity and mortality in survivors of HL. The cumulative incidence of SMs 20 years after completion of therapy has been reported as $10.6 \%$, which increases to $26.3 \%$ at 30 years [2]. Acute leukemia, non-HL and solid tumors including soft tissue sarcomas (STS) are the three main categories of SM [2,3]. While the former two categories are seen often within the initial few years following completion of therapy, the latter predominates a decade following therapy. Depending on length of follow-up and primary treatment modality, STS constitute $8-19 \%$ of all SM cases in HL patients $[3,4]$. Alkylating chemotherapy, radiotherapy, and postulated inherent defects have been proposed as the risk factors for SM development in HL, of which radiotherapy is the major risk factor for STS.

Malignant fibrous histiocytoma (MFH), a form of STS is rare after HL therapy; very few cases have been reported in the adult literature [5-8]. Moreover, most of the post-radiation sarcomas develop more than 10 years following the initial therapy [5-8]. We report a 14 years old autistic male with HL who developed MFH within 3 years of initial HL treatment and 2 years of ASCT and discuss the potential role of genomic instability in early onset SM.

\section{CASE REPORT}

A 14 year old autistic male was diagnosed with nodular sclerosing, stage IV-B HL. At presentation, he had extensive tumor burden with bulky intra-thoracic lymphadenopathy, pulmonary nodules bilaterally and large left pleural effusion, enlarged lymph nodes within the periportal and peripancreatic region, and lesions in the anterior cortex of both kidneys. A positron emission tomography (PET) scan demonstrated numerous foci of abnormal FDG activity corresponding to the findings noted on the contrast enhanced CT scan. Cytogenetic evaluation of the involved lymph node tissue revealed multiple complex clonal abnormalities
(Table I). The bone marrow sample showed normal male karyotype without lymphoma involvement.

The patient received chemotherapy as per Pediatric Oncology Group 9425 protocol consisting of doxorubicin, bleomycin, vincristine, etoposide, prednisolone, and cyclophosphamide (ABVEPC). He did not achieve complete remission after completing a total of seven courses of ABVE-PC chemotherapy. A follow-up PET scan performed after completion of chemotherapy revealed a variable response to treatment with increase in size and intensity of right and left upper lobe lung lesions. In view of primary refractory and progressive disease, he received two cycles of high dose ifosfamide, carboplatin, and etoposide chemotherapy followed by ASCT.

A year following his initial diagnosis, he received carmustine, etoposide, cytarabine, and melphalan, followed by ASCT with $3.20 \times 10^{6} / \mathrm{kg}$ CD $34+$ cells. A follow-up PET scan revealed decreased FDG uptake in most of the tumor, but three foci of abnormal FDG uptake were still seen in the prevascular space in the mediastinum. He received $30 \mathrm{~Gy}$ of radiation to the involved chest fields, after which complete remission of HL was achieved and he had remained asymptomatic for 18 months.

${ }^{1}$ Division of Pediatric Hematology Oncology, Children's Hospital of Michigan, Wayne State University, Detroit, Michigan; ${ }^{2}$ Center for Molecular Medicine and Genetics, Barbara Ann Karmanos Cancer Center, Wayne State University, Michigan; ${ }^{3}$ Department of Pathology, Children's Hospital of Michigan, Wayne State University, Detroit, Michigan; ${ }^{4}$ Department of Radiation Oncology, Barbara Ann Karmanos Cancer Center, Wayne State University, Detroit, Michigan; ${ }^{5}$ Pediatric Blood and Marrow Transplantation, Barbara Ann Karmanos Cancer Center, Wayne State University, Detroit, Michigan

Conflict of interest: Nothing to declare

Dr. Raja Rabah's current address is 2G332 UH, 1500 E. Medical Center Drive, Pediatric Pathology, Department of Pathology, University of Michigan, Ann Arbor, Michigan

*Correspondence to: Süreyya Savaşan, 3901 Beaubien Blvd., Children's Hospital of Michigan, Division of Hematology/ Oncology, Pediatric Blood and Marrow Transplantation, Detroit, Michigan 48201. E-mail: ssavasan@med.wayne.edu

Received 20 June 2010; Revised 21 October 2010; Accepted 2 November 2010 
TABLE I. Karyotypes in Various Tissues

\begin{tabular}{|c|c|c|}
\hline Site & Tissue diagnosis & Karyotype findings \\
\hline Lymph node- at diagnosis & Hodgkin lymphoma & $\begin{array}{l}46, X Y[\mathrm{cp} 18] \\
48, \mathrm{XY},+\mathrm{X}, \operatorname{add}(4)(\mathrm{q} 21),+5,+9, \operatorname{del}(9)(\mathrm{p} 13), \operatorname{add}(12)(\mathrm{q} 24.3) \\
\quad-13, \mathrm{t}(14 ; 15)(\mathrm{q} 10 ; \mathrm{q} 10),+17[\mathrm{cp} 2]\end{array}$ \\
\hline Bone marrow- at diagnosis & Normal & $46, \mathrm{XY}[20]$ \\
\hline Soft tissue mass & MFH & $\begin{array}{l}\text { 46,XY,inv }(6)(\mathrm{p} 21 \mathrm{p} 25)[13] \\
46, \mathrm{XY}, \mathrm{t}(3 ; 13)(\mathrm{q} 12 ; \mathrm{q} 34)[2] \\
46, \mathrm{XY} \text { [nonclonal abnormalities 5] }\end{array}$ \\
\hline $\begin{array}{l}\text { Soft tissue surrounding tumor } \\
\text { resection site }\end{array}$ & $\begin{array}{l}\text { Granulation tissue and foreign } \\
\text { body giant cell reaction }\end{array}$ & 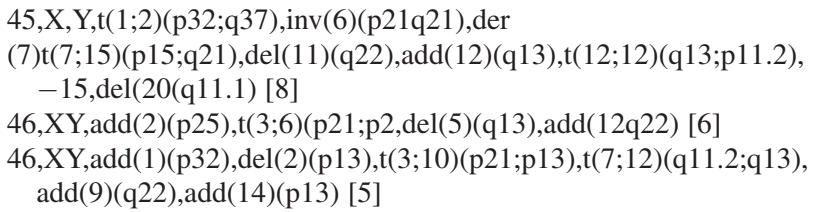 \\
\hline
\end{tabular}

Two years after ASCT, he presented with a $10 \mathrm{~cm} \times$ $8 \mathrm{~cm} \times 6 \mathrm{~cm}$ hard immobile subcutaneous lesion involving the left scapula. The lesion was in the field exposed to prior radiotherapy for HL. On evaluation increased FDG uptake was noted on PET scan. Complete local excision of the lesion was done, which showed inflammatory type of malignant fibrous histiocytoma with anaplasia. In view of malignant nature of the tumor a wide local re-excision was performed, it showed granulation tissue with foreign body reaction without any evidence of tumor. Metastatic work-up was negative. Surgical resection was followed by $60 \mathrm{~Gy}$ of electron beam therapy to the tumor bed. One year after the radiotherapy, the patient continues to be without evidence of HL or MFH.

Cytogenetic analysis on the tissue samples from the two surgical resections revealed two and three different unrelated abnormal clonal tumor cells, respectively (Table I). In view of marked chromosomal aberrations seen in HL, MFH, and granulation tissue, and short interval between initial therapy for HL and development of MFH, spectral karyotyping (SKY) analysis for genomic instability was performed using peripheral blood lymphocytes [9]. According to standard medical genetics practice, clonal chromosomal aberration (CCA) is reported to be present when the same chromosomal aberration is detected at least twice within 20-40 mitotic figures [10]. Based on this practice standard, CCAs refer to common aberrations with over $4-15 \%$ occurrence among the examined cell population. In agreement with this standard, non-clonal chromosome aberration (NCCA) is defined as aberrations with a frequency of less than $4 \%$. In our patient, based on 48 SKY analyzed mitotic figures, there was no CCA detected. However, there was a high level of NCCAs observed pointing at genome instability (Fig. 1) [11-13]. In healthy individuals, cultured lymphocytes show a very low percentage of NCCA, ranging from 0 to $2 \%$ [14]. However, in our patient, the frequency of NCCA was 35\% among examined mitotic figures, of these $15 \%$ were structural NCCA and $20 \%$ were the numerical NCCAs (mainly aneuploidy). Interestingly, there was also high frequency of defective mitotic figures (DMF), which are a type of structural NCCA that is also associated with genome instability [11-13]. Based on the examination of 189 Giemsastained mitotic figures, the DMF frequency was very high at $15 \%[12,13]$.

Pediatr Blood Cancer DOI 10.1002/pbc

\section{DISCUSSION}

Malignant fibrous histiocytoma, a type of STS has been reported following radiation therapy in HL [5-8]. Though the absolute risk of developing MFH is not known, relative risk (RR) of developing STS is the highest among all SM (RR-135) $[3,4]$. Multiple unrelated clonal abnormalities are not uncommon
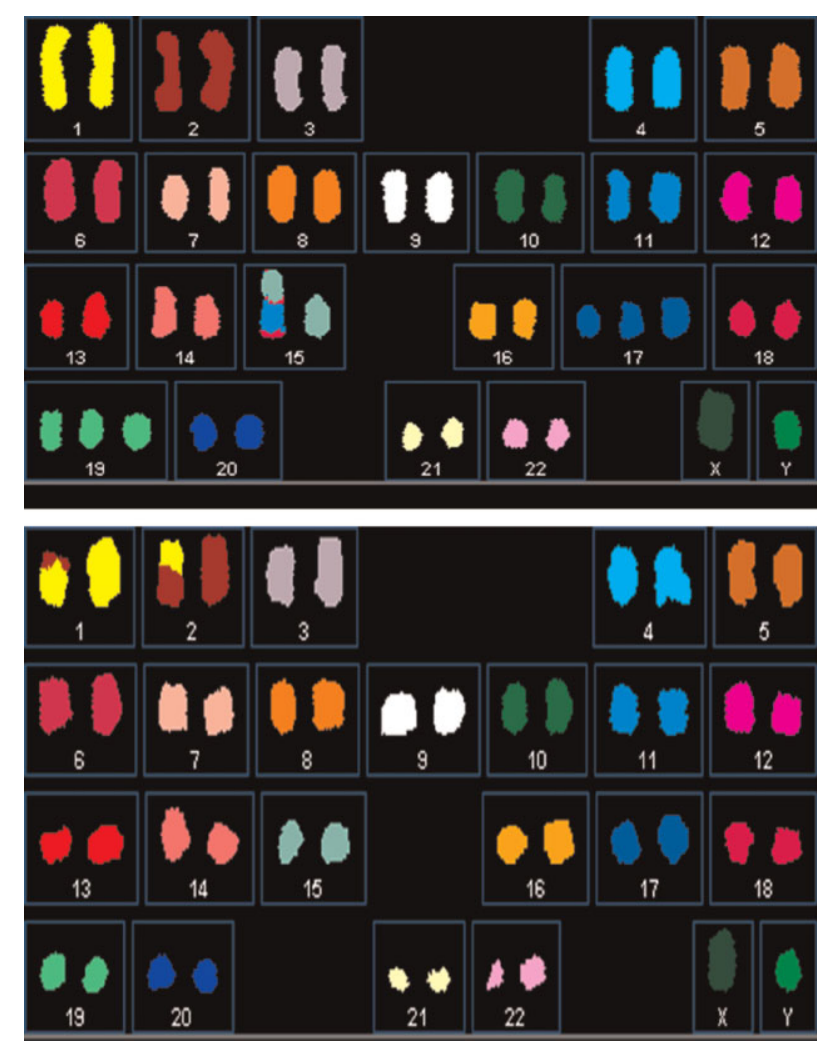

Fig. 1. Representative examples of NCCA. Upper image shows $\mathrm{t}(15 ; 11 ; 18)$ and three copies of chromosome 17 and 19. Lower image shows the translocation between chromosome 1 and 2 . [Color figure can be viewed in the online issue, which is available at wileyonlinelibrary. com.] 
in STS, it has been documented to occur in three out of the ten cases of post radiation sarcomas reported by Mertens et al. [15]. Most cases of MFH have been reported to occur in adults (18-59 years) who have received more than $40 \mathrm{~Gy}$ (40-53 Gy) of radiotherapy and most of them developed MFH after a latent period of more than 10 years [5-8]. The shortest latency period reported in a series of post-radiation sarcoma cases in HL was four years [5]. In our patient, despite smaller radiation dose (30 Gy), MFH developed within 18 months of RT and 34 months of initial therapy, which is very unusual. High dose chemotherapy and ASCT are known to increase the risk of SM [5,8]. Presence of multiple unrelated clonal karyotype abnormalities in both MFH and granulation tissue and very short latency prompted us to investigate for underlying predisposition to malignancy in our case. Although there was no tumor tissue identified by microscopy, there is a possibility of contaminating malignant cells among the reactive tissue cells.

Multiple non-specific numerical and structural aberrations of chromosomes have been well documented in HL [16]. Heng et al. have recently proposed that genomic instability leading to NCCA aberrations might be the main factor in malignant transformation and progression in a majority of cancers [12,17]. NCCA is a newly identified marker of overall genome instability [11-13]. The frequencies of NCCA have been directly linked to tumorigenicity [18]. In normal individuals, the frequency of NCCA in their cultured blood lymphocytes is between 0 and $2 \%$ [14]. The elevated frequency of NCCA (35\%) detected in our case suggests high level genome instability. However, it is not clear how much of this high-level of instability is caused by recent exposure to chemotherapy or from the individual's inherent chromosomal instability. Nevertheless, further radiation exposure to the local tissue with already known karyotype abnormalities can increase the risk of other SM in our case.

Multiple loci of chromosomal instability (16p11.2, 15q13, and $7 q$ ) and increased incidence of PTEN mutations have been identified in a small percentage of children with autism $[19,20]$. To date, there have been no studies to see whether the genomic instability noted in these patients are associated with increased NCCA, which if present, might predispose them to the development of malignancy. In our case, though the SKY results were very abnormal, the initial karyotype done at the time of diagnosis was normal by classical cytogenetics. Further investigation specifically looking for known loci of chromosomal instability in autism was not performed. In future more studies are needed to better understand the possible association.

In conclusion, the development of MFH as an SM much earlier than normally seen in survivors of HL is very unusual. The presence of multiple clonal cytogenetic abnormalities in tumor and reactive tissues, and further demonstration of high-level NCCA in peripheral blood lymphocytes suggest genome instability in our patient.

\section{REFERENCES}

1. Hunger SP, Link MP, Donaldson SS. ABVD/MOPP and lowdose involved-field radiotherapy in pediatric Hodgkin's disease: The Stanford experience. J Clin Oncol 1994;12:2160-2166.
2. Bhatia S, Yasui Y, Robison LL, et al. High risk of subsequent neoplasms continues with extended follow-up of childhood Hodgkin's disease: Report from the Late Effects Study Group. J Clin Oncol 2003;21:4386-4394.

3. Mauch PM, Kalish LA, Marcus KC, et al. Second malignancies after treatment for laparotomy staged IA-IIIB Hodgkin's disease: Long-term analysis of risk factors and outcome. Blood 1996;87: $3625-3632$.

4. Wolden SL, Lamborn KR, Cleary SF, et al. Second cancers following pediatric Hodgkin's disease. J Clin Oncol 1998;16: 536-544.

5. Smith J. Postradiation sarcoma of bone in Hodgkin disease. Skeletal Radiol 1987;16:524-532.

6. Griesser GH, Hansmann ML. Soft tissue sarcoma as second malignant lesion after therapy for Hodgkin's disease. Report of two cases and review of the literature. J Cancer Res Clin Oncol 1985;110:238-243.

7. Nonaka M, Kadokura M, Ohkubo F, et al. Post radiation inflammatory malignant fibrous histiocytoma arising from the chest wall. Ann Thorac Cardiovasc Surg 2001;7:371-374.

8. Oddou S, Vey N, Viens P, et al. Second neoplasms following high-dose chemotherapy and autologous stem cell transplantation for malignant lymphomas: A report of six cases in a cohort of 171 patients from a single institution. Leuk Lymphoma 1998;31:187-194.

9. Heng HH, Ye CJ, Yang F, et al. Analysis of marker or complex chromosomal rearrangements present in pre- and post-natal karyotypes utilizing a combination of G-banding, spectral karyotyping and fluorescence in situ hybridization. Clin Genet 2003;63: 358-367.

10. ISCN, Shaffer LG SM, Campbell, LJ editors. An international system for human cytogenetic nomenclature. Switzerland: S. Karger AG; 2009, 89p.

11. Heng HH, Bremer SW, Stevens J, et al. Cancer progression by non-clonal chromosome aberrations. J Cell Biochem 2006; 98:1424-1435.

12. Heng HH, Stevens JB, Liu G, et al. Stochastic cancer progression driven by non-clonal chromosome aberrations. J Cell Physiol 2006;208:461-472.

13. Ye CJ, Liu G, Bremer SW, et al. The dynamics of cancer chromosomes and genomes. Cytogenet Genome Res 2007;118: 237-246.

14. Carrano AV, Natarajan AT. International Commission for Protection Against Environmental Mutagens and Carcinogens. ICPEMC publication no. 14. Considerations for population monitoring using cytogenetic techniques. Mutat Res 1988;204: 379-406.

15. Mertens F, Larramendy M, Gustavsson A, et al. Radiationassociated sarcomas are characterized by complex karyotypes with frequent rearrangements of chromosome arm $3 p$. Cancer Genet Cytogenet 2000;116:89-96.

16. Re D, Zander T, Diehl V, et al. Genetic instability in Hodgkin's lymphoma. Ann Oncol 2002;13:19-22.

17. Heng HH, Stevens JB, Bremer SW, et al. The evolutionary mechanism of cancer. J Cell Biochem 2010;109:1072-1084.

18. Ye CJ, Stevens JB, Liu G, et al. Genome based cell population heterogeneity promotes tumorigenicity: The evolutionary mechanism of cancer. J Cell Physiol 2009;219:288-300.

19. Eichler EE, Zimmerman AW. A hot spot of genetic instability in autism. N Engl J Med 2008;358:737-739.

20. Butler MG, Dasouki MJ, Zhou XP, et al. Subset of individuals with autism spectrum disorders and extreme macrocephaly associated with germline PTEN tumour suppressor gene mutations. J Med Genet 2005;42:318-321. 